\title{
Improved Fitness Proportionate Selection-Based Genetic Algorithm
}

\author{
Yu Fengrui ${ }^{1, a}, F u$ Xueliang ${ }^{1, b^{*}}$, Li Honghui ${ }^{1, c}$ and Dong Gaifang ${ }^{1, d}$ \\ ${ }^{a}$ College of Co mputer Science and Information Engineering, Inner Mongolia Agricultural University , \\ P.R. China

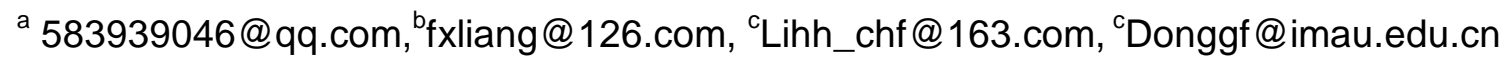 \\ ${ }^{*}$ Corresponding Author: Fu Xueliang
}

\begin{abstract}
Keywords: genetic algorithm $(\mathrm{GA})$, fitness proportionate selection, roulette wheel selection, travelling salesman problem (TSP)

Abstract. Genetic algorithms are typical swarm intelligence techniques based on the mechanics of natural selection and natural genetics, which combines artificial survival of the fittest concept with genetic operations abstracted from nature. Since the genetic algorithm has good global search capability, as well as the parallel nature of other advantages, it has been widely used in combinatorial optimization, machine learning, signal processing field, adaptive control and artificial life and so on. It is one of the key technologies related to modern intelligent calculation. Fitness proportionate selection, as a common selection method for GA, is usually implemented with method of roulette wheel selection. In this paper, An improved selection method based on fitness proportionate selection was presented. Computational results show that the method which proposed in this paper improved the result precision and better astringency by solving TSP problem.
\end{abstract}

\section{Introduction}

Adaptation in Nature and Artificial System was published by Professor Holland in 1970s, systematically elaborating basic theories and methods of genetic algorithm, presenting principle theorem-schema theorem of GA, and laying the theoretical basis for GA development [1]. During 1980s, GA was widely applies in different fields. Operators of standard GA generally covered three basic forms, selection, cross and variation, which constituted the core with powerful searching ability of GA [2]. Selection is the major carrier for the simulations of life reproduction and survival of the fittest in the natural world. Selection refers to the process of selecting individuals with high fitness value in current group to generate a mating pool. Recently, there are forms, including fitness proportionate selection, Boltzmann selection, ranking selection, tournament selection and elitist selection [3]. Fitness proportionate selection, as a basic selection model, is always a common selection model of GA and is usually implemented in the model of roulette wheel selection.

Travelling Salesman Problem (TSP) is a classical combinational optimization problem as well as a NP-hard problem [4]. The history was long and related studies had achieved great progressions, yet the problem is not thoroughly solved. With the increasing scale of cities, optional route amount increases in exponential orders. As the exact optimal solution is generally difficult to be found, it is important to find an effective approximate solution algorithm. Because GA is not restrained by restrictive hypothesis concerning searching space without requirements on continuity, derivability and single-peak and there are features including global optimization, implicit parallelism and robustness [5], the method shows favorable performances and results when solving complicated problems, such as combinational optimization, pattern recognition and computer network optimization, which are difficult to be solved with traditional methods. GA has been applied by people to solve large-scale TSPs [6], and some satisfactory results have been achieved.

In this paper, we still take the typical TSPs problem as the research object, through the analysis of the running time and the number of satisfactory solutions of Genetic Algorithm and the optimization algorithm, we give the performance evaluation of the improved algorithm. 


\section{Fitness Proportionate Selection Mode and Optimization Method}

For the Fitness Proportionate Selection Mode, fitness values of all individuals were calculated firstly. The proportion of the fitness in the total group fitness was then calculated, representing the probability of the indiviciual being selected during the selection process. For a group with a given scale of $\mathrm{n}, p=\left\{a_{1}, a_{2}, \ldots, a_{n}\right\}$ and the fitness of individual is $f\left(a_{i}\right), a_{i} \in p$, so we can calculate the selection probability of individual fitness by formula (1).

$$
p_{s}\left(a_{j}\right)=\frac{f\left(a_{j}\right)}{\sum_{i=1}^{n} f\left(a_{j}\right)}, j=1,2, \ldots, n ;
$$

The above formula determines probability distributions of individuals from the offspring population, among which expected individual survival amount in the population of the parent generation is $p\left(a_{j}\right)=n p, j=1,2, \ldots, n$. The global idea of this selection mode is to make individuals with high fitness breed more and make individuals with low fitness breed less and even no breed. Generally, the scale of individual from the parent generation equals to that from the offspring generation. Calculated expected amount $p\left(a_{i}\right)$ is generally not an integer. If rounding-off is adopted, the population scale is usually changed after summation of individuals from the offspring population. The roulette wheel method is then for this issue.

\section{Roulette Wheel Selection}

The cumulative probability is calculated with selection probabilities of all individuals. The cumulative probability of the no. $\mathrm{k}$ individual can be calculated by formule (2).

$$
p_{x}\left(a_{k}\right)=\sum_{j=1}^{k} p_{x}\left(a_{j}\right)
$$

Then random number e, which is between 0 and 1 , is generated and compare with $p_{x}\left(a_{k}\right)$ to determine selection individual. If $a_{k-1}<e<a_{k}$, the no. $\mathrm{k}$ individual is selected. Repeated $\mathrm{n}$ rounds are conducted to generate $\mathrm{n}$ individuals of offspring generation.

\section{Optimization Selection Method}

Expected survival amount of individuals $p\left(a_{j}\right)$ is directed down rounded to achieve $x\left(a_{j}\right)$. The summation of $x\left(a_{j}\right)$ is calculated by formula (3).

$$
m=\sum_{j=1}^{n} x\left(a_{j}\right)
$$

Then, (n-m) individuals should be generated to form an intact offspring generation. Expected survival amounts $p\left(a_{j}\right)$ of all individuals are ranked in descending order. The top (n-m) individuals are selected to achieve an intact offspring generation. For example, the no. $\mathrm{k}$ individual ${ }^{a_{k}}$ is in the top (n-m) individuals, the actual selection amount of the no. $\mathrm{k}$ individual in the offspring generation is $\left(x\left(a_{k}\right)+1\right)$.

\section{Comparison between the Algorithms before and after Optimization}

(1) For the Roulette Wheel Method, selection is made completely depending on random numbers, which increases uncertainty of selection. It could be said that the optimization method in this paper is the most ideal performance for the Roulette Wheel Method;

(2) For a population scale of $n=50$, the average selection probability is only 0.02 . When the scale of a population increases further, the selection probability of even an optimal individual is very low. For the Roulette Wheel Method, the randomness might cause losses of excellent individuals. The optimization method in this paper reserves excellent individuals and prevents losses of good solutions.

(3) With the Roulette Wheel Method, individuals of the offspring generation do not conduct selection completely according to fitness of individuals of the parent generation, while the optimization method in this paper does. It fully 
shows the situation of more reproduction in adapters and less reproduction and even no reproduction in non-adapters, which facilitates convergence.

\section{Implementation and experimental results}

The mechanism adopted in this paper for solving TSP with GA was self-adapting cross and self-adapting mutation operator $^{[7-8]}$, and the strategy of retaining optimal values after crosses and mutations of every generation was adopted ${ }^{[9]}$. With the improved mechanism, impacts of different selection modes on GA could be better reflected. The algorithm of GA iterative process is illustrated in figure 1.

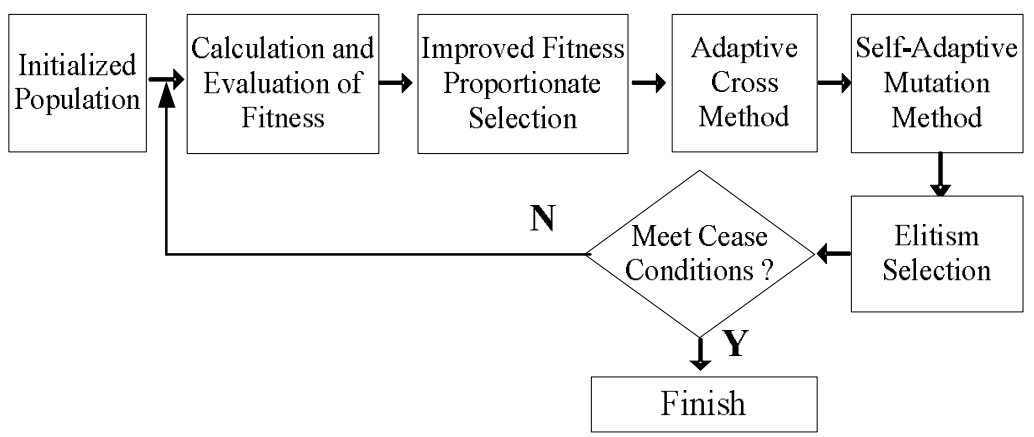

Figure 1 Flow of the GA algorithm

The GA, Optimization GA are implemented using C++. For GA, Roulette wheel method isused to conduct the selection of individuals and the Optimization GA is combined with the improved method to conduct the selection of individuals.

We selected the following three benchmark TSPs for our experiment: bayg29, att48, ch130 and a280. The parameters used for individual problems are given in Table 1, and the maximum number of iteration is set to 500 for all cases. For GA and Optimization GA, the size of population is as same as the number of cities whereas the number of genes for each individual. Each case is repeated for 50 times for data analysis and all simulations are conducted on the same PC to make our comparison on the same ground later.

Table 1. Experimental results for TSPs using GA and Optimization GA (50 runs for each case)

\begin{tabular}{c|c|c|c|c|c}
\hline \multirow{2}{*}{ TSP } & \multicolumn{2}{|c|}{ GA } & \multicolumn{2}{c|}{ Optimization GA } & \multirow{2}{*}{$\begin{array}{c}\text { Known Optimal } \\
\text { Solution }\end{array}$} \\
\cline { 2 - 5 } & Best & Mean & Best & Mean & 1610 \\
\hline Byag29 & 3302 & 3378 & 3169 & 3210 & 10628 \\
att48 & 15628 & 16042 & 14906 & 15207 & 6110 \\
ch130 & 9784 & 9914 & 9512 & 9625 & 2579 \\
\hline
\end{tabular}

Table 1 shows the statistics of our experimental results in terms of accuracy of the best solutions. The known optimal solutions are taken from the TSP Library (TSPLIB[20]). After 500 iterations for all cases, surprisingly, the efficiency 
of the Optimization GA algorithm to find the optimal solution is significantly improved.

Table 2. Running time and iteration number of GA and Optimization GA (50 runs for each case)

\begin{tabular}{c|c|c|c|c|c|c|c|c}
\hline \multirow{2}{*}{ TSP } & \multicolumn{4}{|c|}{ GA } & \multicolumn{4}{c}{ Optimization GA } \\
\cline { 2 - 8 } & $\begin{array}{c}\text { Best } \\
\text { time }\end{array}$ & $\begin{array}{c}\text { Mean time } \\
(50)\end{array}$ & $\begin{array}{c}\text { Best } \\
\text { iteration } \\
\text { number }\end{array}$ & $\begin{array}{c}\text { Mean iteration } \\
\text { number } \\
(50)\end{array}$ & $\begin{array}{c}\text { Best } \\
\text { time }\end{array}$ & $\begin{array}{c}\text { Mean time } \\
(50)\end{array}$ & $\begin{array}{c}\text { Best } \\
\text { iteration } \\
\text { number }\end{array}$ & $\begin{array}{c}\text { Mean iteration } \\
\text { number } \\
(50)\end{array}$ \\
\hline Byag29 & 309 & 347 & 278 & 301 & 386 & 547 & 268 & 422 \\
att48 & 421 & 512 & 364 & 421 & 448 & 627 & 337 & 589 \\
ch130 & 720 & 849 & 635 & 756 & 490 & 593 & 456 & 588 \\
\hline
\end{tabular}

Table 2 shows the statistics of our experimental results in operation time and operation iteration number. It could be viewed that both operation times and iteration numbers in the Optimination GA were superior to the GA after 500 iterations for all cases.

\section{Conclusion}

Fitness proportionate selection, as a common selection method for GA, is usually implemented with method of roulette wheel selection. The roulette wheel selection mode was improved in this paper to make the selection mode better accord with the rule of "survival of the fittest". Results of experiment, which was conducted by solving TSP, verified the validity of this method, which improved result precision and facilitated convergence of the algorithm. The selection method in this paper was a supplementary method to fitness proportionate selection. As GA is an optimization algorithm with randomness, selection between these two methods should be conducted according to practical optimized problems.

\section{Acknowledgements}

This work was financially supported by the National Natural Science Foundation of China (61063004,61363016), the Natural Science Foundation of Inner Mongolia Autonomus Region (2015MS0626,2015MS0605), and The key project of Scientific research in Inner Mongolia Autonomous Region Colleges and Universities (NZJJ14100).

\section{References}

[1] J.H. Holland. Adaptation in Natural and Artificial Systems. The University of Michigan Press. Ann. Arbor. 1975.

[2] De Jong, K.A.An analysis of the behavior of a class of genetic adaptive systems [M] .University of Mi chigan, No.79 -9381, 1975

[3]. D Applexgate, R Bixby, et al. Implementing the Danting-Fulkerman-Johnson Algorithm for Large Traveling Salesman Problems. Mathematical Programming. 2003, 97(1-2). 91-98.

[4] LI Min-qiang, KOU Ji-song et al., Basic Theory and Application of Genetic Algorithm. Beijing: Science Press. 2002.34-50

[5] Dong G, Fu X, Xue H. An Ant System-Assisted Genetic Algorithm For Solving The Traveling Salesman Problem[J]. International Journal of Advancements in Computing Technology, 2012, 4(5):165-171.

[6] Rafiei H, Rabbani M, Gholizadeh H, et al. A novel hybrid SA/GA algorithm for solving an integrated cell formation-job scheduling problem with sequence-dependent set-up times[J]. International Journal of Management Science \& Engineering Management, 2015:1-9. 
[7] Liu L L, Hu R S, Hu X P, et al. A hybrid PSO-GA algorithm for job shop scheduling in machine tool production[J]. International Journal of Production Research, 2015:1-27.

[8] Umbarkar A J, Sheth P D, Babar S V. Solving 0/1 Knapsack Problem Using Hybrid TLBO-GA Algorithm[J]. Advances in Intelligent Systems \& Computing, 2015, 335:1-10.

[9] Wu L, Yang X. Optimization of extrusion die of spherical plain bearings based on bp neural network and ga algorithm[J]. Zhongguo Jixie Gongcheng/china Mechanical Engineering, 2015, 26(10):1351-1355.

[10] Gao Mingfang, Fu Xueliang, Dong Gaifang, Li Honghui. An Adaptive Mutation Multi-particle Swarm Optimization for Traveling Salesman Problem, 3rd International Conference on Material, Mechanical and Manufacturing Engineering (IC3ME 2015).2015:1003-1007. 